\title{
Uveitis induced cyclospasm induced myopia-a unique
}

\section{case}

\section{Introduction}

The near response consists of a triad of normal accommodation, convergence and miosis. If one or more of these components exceeds the required demand, there occurs a spasm of near reflex or spasm of accommodation. Spasm of accommodation or accommodative spasm (AS) is a rare condition characterized by intermittent and usually painful convergence, accommodation, miosis and myopic shift in varying combinations. ${ }^{1}$ With AS, hyperopes may appear less hyperopic, emmetropes may appear myopic, and myopes may appear more myopic. ${ }^{2,3} \mathrm{AS}$ is rare and occurs in less than $3 \%$ of patients with accommodative disorders. ${ }^{4}$ Even though uveitis is known to produce ciliary muscle spasm, there has not been any case in literature of uveitis induced AS with induced myopia as a presenting symptom. This case report documents for the first time, the occurrence of such a rare condition i.e., uveitis induced accommodative spasm induced myopia.

\section{Case report}

A 34 year old Indian male presented to our OPD with complaints of pain and redness, followed by blurring of distant vision in right eye since 1 month. Past ocular history included use of Prednisolone acetate eye drop $1 \%$ three times daily, in right eye. The patient had neither similar history in the past nor had a history of trauma. He had never worn glasses earlier, and claimed to have had clear uncorrected vision 1 month prior to the problems. He had no other comorbidities and was not on any other medications. Presenting unaided visual acuity was $20 / 80$ which improved to $20 / 20$ with $-1.25 \mathrm{D} \mathrm{Sph} /-0.50 \mathrm{D}$ Cyl x 100 degrees in the right eye. Versions and ductions were full, as were pupillary response to light. Contrast sensitivity was 1.5 in the right eye and 1.65 in the left eye. The near point of convergence (NPC) and near point of accommodation (NPA) was measured using Royal Air force ruler. The NPC was $10 \mathrm{~cm}$ and NPA was $14 \mathrm{~cm}$. The positive relative accommodation was measured and was found to be $-6.75 \mathrm{D} \mathrm{Sph}$.

Anterior segment examination using slit-lamp showed pigments in corneal endothelium, grade 2 cells and flare in the anterior chamber. The pupil was miotic. Anterior 1/3rd of vitreous had $1+$ cells. Dilated indirect ophthalmoscopy findings were normal. Dilated retinoscopy with Tropicamide was $+0.25 /+0.75 / 90 / 180$ and subjective refraction was $-1.25 \mathrm{D} \mathrm{Sph} /-0.50 \mathrm{D}$ Cyl x 100 degrees which improved the visual acuity to 20/20. Additional investigations were performed to rule out any retinal pathology, which included Optical Coherence Tomography (OCT) which showed no significant retinal edema. Suspecting this to be cyclospasm induced myopia, the patient was advised to come after three days of atropinization with $1 \%$ atropine eye ointment TID per day, along with Prednisolone acetate 1\% eye drop for eight times. On the fourth day, unaided visual acuity remained the same and dilated retinoscopy obtained was $+2.25 /+1.50 / 90 / 180$. Thus we could demonstrate a hyperopic shift with three days of atropinization. The patient was then advised to continue atropine eye ointment for ten more days and taper Prednisolone acetate eye drop to 4 times, then three times, then two times for 1 week each, and to review after
Volume 7 Issue 3 - 2017

\author{
Gopal S Pillai,Affna Tajudeen \\ Amrita Institute of Medical Sciences, Amrita \\ vishawavidayapeetham, Kochi, India
}

Correspondence: Gopal S Pillai, HOD Department of Ophthalmology,Amrita Institute of Medical sciences, Amrita Vishwavidyapeethom, Kochi, Kerala, India, Tel 04842851099 , Email gopalspillai@gmail.com

Received: July 31, 2017 | Published: August 16, 2017

three weeks. After three weeks, unaided visual acuity improved to $20 / 60$. The retinoscopy was repeated by the same examiner and the reading was almost same as before, ie,. $+2.00 /+1.50 / 90 / 180$. The patient was now asked to stop atropine and Prednisolone acetate eye drop after tapering it. He was asked to come after two days of stopping it, so as to repeat the subjective refraction and to repeat the near point of accommodation and convergence, and positive relative accommodation. The patient came after 3 weeks of stopping atropine. On this day, the unaided visual acuity was $20 / 20$, NPC was $11 \mathrm{~cm}$ and the NPA was $18 \mathrm{~cm}$. The PRA was also repeated and was found to be -3.00 DSph. The patient was asked to follow up after a month when he was maintaining 20/20 vision. He is asked to come if the symptoms recurred.

\section{Discussion}

Anterior uveitis is the commonest form of intraocular inflammation which involves inflammation of the iris alone (iritis), anterior part of ciliary body (anterior cyclitis) or both structures (iridocyclitis). ${ }^{5}$ Ciliary spasm can cause blurred vision in anterior uveitis, ${ }^{6}$ which is a condition where there is an involuntary, sustained accommodation in the absence of an accommodative stimulus. ${ }^{7}$ This case report is the first documentation of accommodative spasm induced myopia as a result of anterior uveitis. Usually acute anterior uveitis presents with pain, redness, photophobia and mild blurring of vision. Decrease of distant vision is usually mild and vision is usually to the tunes of $6 / 6$ to 6/9. We may suspect additional cystoid macular edema or optic neuritis associated with acute anterior uveitis if vision decreases further. In most cases in which initial treatment with cycloplegics are undertaken, the patients may complain of decrease in near vision only. Our case presented with decrease of distant vision with normal near vision, he gave no history of myopia or glasses in the past and normal uncorrected vision before the redness started. His refraction demonstrated myopia and we suspected AS at that point of time. Hence we measured the positive relative accommodation and found that it is very high, confirming the diagnosis of AS induced myopia. With weak cycloplegic like tropicamide, there was no change in the degree of myopia, but after atropinization, the induced myopia started reducing. Once AS was controlled, we stopped the atropine and his myopia completely reversed. His positive relative accommodation came back to normal values. 
Even though there are case reports related to AS in siblings and AS in patients with exotropia, there is no similar case reported earlier, describing uveitis induced accommodative spasm induced myopia. Patients with uveitis who present with complaints of blurring of distant vision may undergo certain test like measuring NPA, NPC and PRA, so that one does not miss the actual amount of refractive error at the time of presentation. Prescription of usual cycloplegics like tropicamide may not reverse the spasm and stronger cycloplegics like atropine may need to be used in these conditions. Atropine causes maximum relaxation of ciliary muscle thereby subsiding or eliminating the amount of myopia. The excessive accommodative tone usually causes pseudomyopia, ${ }^{8}$ which seems probable in this case.

\section{Acknowledgements}

None.

\section{Conflicts of interest}

There are no conflicts of interest.

\section{Funding}

None.

\section{References}

1. Cogan DG, Freese CG. Spasm of the Near Reflex. Arch Ophthalmol. 1955;54:752-759.

2. London R, Wick B, Kirschen D. Post-traumatic pseudomyopia Optometry. 2003;74:111-120.

3. Moore S, Stockbridge L. Another approach to treatment of accommodative spasm. Am Orthopt J. 1973;23:71-72.

4. Daum KM. Accommodative dysfunction. Doc Ophthalmol. 1983;55(3):177-198.

5. Jabs DA, Nussenblatt RB, Rosenbaum JT. Standardization of uveitis nomenclature (SUN) for reporting clinical data. -Results of the First International Workshop. Am J Ophthalmol. 2005;140(3):509-516.

6. Esra Guney, Ilknur Tugal-Tutkun. Symptoms and signs of anterior uveitis. Uveitis. US Ophthalmic Review. 2013;6(1):33-37.

7. Baikoff G, Lutun E, Ferraz C, et al. Static and dynamic analysis of the anterior segment with Optical Coherence Tomography. J Cataract Refract Surg. 2004;30(9):1843-1850.

8. Smith JL. Accommodative spasm versus spasms of the near reflex. $J$ Clin Neuro-Ophthalmol. 1987;7:132-134. 